\title{
Connectedness of the Fibers of a Liouvillian Function
}

By

Emmanuel PAUL*

\begin{abstract}
Let $D$ be a normal crossing divisor in a complex analytic manifold of dimension $n$, and let $\Omega$ be a closed logarithmic one-form, with poles on $D$. Under appropriate hypothesis, we prove the connectedness of the fibers for a primitive of $\Omega$ in "good" neighborhoods of $D$. We deduce the connectedness of the fibers of Liouvillian functions of type $f=f_{1}^{\lambda_{1}} \cdots f_{p}^{\lambda_{p}}$ at the origin of $C^{n}$, under two conditions: the first extends the usual notion that " $f$ is not a power". The second excludes certain meromorphic functions.
\end{abstract}

\section{Introduction}

We consider a germ at the origin of $C^{n}$ of a Liouvillian function of the type:

$$
f=f_{1}^{\lambda_{1}} \cdots f_{p}^{\lambda_{p}},
$$

where the $f_{i}$ are germs of analytic irreducible functions, and the $\lambda_{i}$ are non-zero complex numbers. We say that such a function "is not a power" if the value 1 belongs to the additive group generated by the exponents $\lambda_{i}$. This definition generalizes that for germs of holomorphic functions. The "values" of this multivalued function belong to the quotient of $C^{*}$ by the multiplicative group generated by the exponentials of the numbers $2 i \pi \lambda_{j}$. This allow us to define the regular fibers (a priori with several connected components) of this function. The aim of this article is to prove the following result:

Theorem A. Let $f=f_{1}^{\lambda_{1}} \cdots f_{p}^{\lambda_{p}}$ be a germ of a Liouvillian function which is not a power. Suppose that the complex numbers $\lambda_{i}$ are not related by relations

Communicated by T. Kawai, December 24, 1996.

1991 Mathematics Subject Classification(s): 32S65, 34A20.

* Laboratoire Emile Picard, U.M.R. C.N.R.S. 5580

Université Paul Sabatier, 31062 Toulouse Cedex 4, France. 
with positive integer coefficients, and that the ratios of elements of the form $\sum n_{i} \lambda_{i}$, where the $n_{i}$ are positive integers, are not negative real numbers. Then there exists a fundamental system of neighborhoods of the origin in which the fibers of a representative of this function are connected.

This result was already known for germs of holomorphic functions. In the case of dimension 2, it was proved in [1] and [8]. The case of dimension $n$ can be reduced to that of dimension 2 by an argument using hyperplane sections of LÊE Dung Trang in [7]. Theorem A extends this result to the multivalued context, for any dimension. The main difficulty here is the absence of a Milnor fibration. This fibration may be replaced by the existence of a transverse flow ([6] and [11]), which can be used to define and to study vanishing cycles on the fibers of such a function (for dimension 2: see [11]). Nevertheless, the problem of the connectedness of the fibers has been open up until now.

We remark that among the Liouvillian functions for which there exists a ratio of elements $\sum n_{i} \lambda_{i}$ which is real and negative, we can find elements which do not satisfy the property of connectedness of the fibers: in the last part of this article, we describe a family of counter-examples. Among them, we find some meromorphic functions. The condition on the ratios of elements $\sum n_{i} \lambda_{i}$ is not optimal. It can be improved - see Remark (1.4) - by requiring that only ratios of some elements of the form $\sum n_{i} \lambda_{i}$ (given by a desingularization process) are not negative real numbers.

Our strategy is first to prove Theorem A for a function of the form $z_{1}^{\lambda_{1}} \cdots z_{p}^{\lambda_{p}}$, where the $z_{i}$ are coordinates at the origin of $C^{p}$. Then we use a desingularization of the map $f_{1} \cdots f_{p}$ to prove the general case: the first case is a local model of the global situation obtained in the neighborhood of an exceptional divisor. It is easy to understand how the local connected components can be glued together in a neighborhood of an irreducible component of this divisor. On the other hand, to get the global result along this divisor, one must use a tool introduced in [9]: a "Clemens structure" adapted to the closed logarithmic form $d f / f$. This structure gives us local transverse coordinates adapted to the logarithmic form, which furthermore satisfy certain compatibility conditions when we change strata in the divisor. We obtain the following result: 
Theorem B. Let $\Omega$ be a closed logarithmic form with poles on a normal crossing divisor $D$ in a complex analytic manifold $M$ of dimension $n$. Suppose that the residues of $\Omega$ are non-vanishing, and that for any pair of irreducible components of $D$ with non-empty intersection, the ratio of the corresponding residues are not negative real numbers. Let $F$ be a primitive of $\Omega$. Then there exists a fundamental system of neighborhoods of $D$ in $M$ in which the fibers of $F$ are connected.

Let us now indicate the main motivation of this work. Apart from the natural idea to extend the singularity theory of single valued maps to a class of multivalued ones, we may also consider the point of view of ordinary differential equations. In dimension 2 , notice that $f_{1}^{\lambda_{1}} \cdots f_{p}^{\lambda_{p}}$ is a first integral for a germ of holomorphic one-form, whose type is called "non-dicritical generalized curve" by C. Camacho, A. Lins Neto and P. Sad in [2]: These forms allow a desingularization without "dicritical components", and without "saddle-node" singularities. One cannot hope to be able to describe the topology of all generalized curves. Nevertheless, this description is more reasonable for the restricted class of one-forms with "solvable holonomy". The case we treat is the first step in this direction: we study the 0-homology of a generalized curve with abelian linearizable holonomy.

The "holonomy of a germ of a one-form" is the usual holonomy of each component of the exceptional divisor (which in the non-dicritical case are leaves) after a reduction process. It is defined by analytic extension of a transverse local coordinate $z$ which is a local first integral at a regular point of the foliation. This idea is not sufficient to understand all the transverse structure along the divisor: in particular, it does not describe how the semi-global foliations in a neighborhood of each component are glued together. For this reason, we introduced in ([12]) a "generalized holonomy" acting on all the "holomorphic" or "linearizable" area of the divisor: the local transverse first integral $z$ is replaced by a first integral on this area of the form $f_{1}^{\lambda_{1}} \cdots f_{p}^{\lambda_{p}}$, factorizing all the other first integrals, that is to say, with connected fibers. This gives another motivation to characterize them.

\section{§1. Reduction of the Problem}

Let $U$ be an open set. We choose representatives of the germs $f_{i}$ on $U$ such that the homology of $U \backslash\left(f_{1} \cdots f_{p}=0\right)$ is independent of $U$. Then the group $H$ of periods of the closed form $\Omega=\sum_{i=1}^{p} \lambda_{i}^{d f_{i}}$ is also independent of $U$, 
and is generated by the numbers $2 i \pi \lambda_{j}, j=1, \cdots, p$. The fibers of a function $f=f_{1}^{\lambda_{1}} \cdots f_{p}^{\lambda_{p}}$ are defined in the following way: $f$ is the exponential of $F$, where $F=\sum_{i=1}^{p} \lambda_{i} \log f_{i}$ is a multivalued primitive of $\Omega$. This primitive is a single valued map taking its values in the quotient $C / H$. So $f_{1}^{\lambda_{1}} \cdots f_{p}^{\lambda_{p}}$ is single valued in the quotient $C^{*} / G$ where $G$ is the multiplicative group generated by the numbers $\exp 2 i \pi \lambda_{j}$. We call fibers of $f$ those of this map $F:(\exp F)^{-1}(c)$, $c \in C^{*} / G$.

Remark 1.1. If the value 1 belongs to the subgroup of $(C,+)$ generated by $\lambda_{j}$, then the exponential map: $C / H \rightarrow C^{*} / G$ is bijective.

From this elementary remark, we deduce that if $f_{1}^{\lambda_{1}} \cdots f_{p}^{\lambda_{p}}$ is not a power, then the fibers of $f_{1}^{\lambda_{1}} \cdots f_{p}^{\lambda_{p}}$ are those of the primitive $F=\sum_{i=1}^{p} \lambda_{i} \log f_{i}$. It thus suffice to study the connectedness of the fibers of $F$.

We now make clear the ideas introduced in the statement of Theorem B. Recall first (see[13]) that a meromorphic form $\Omega$, with poles on a divisor $D$ in a complex analytic manifold $M$, is logarithmic if at any point of $D$ with local reduced equation $h=0$, the forms $h \Omega$ and $h d \Omega$ are holomorphic.

We now consider a closed logarithmic form with poles on $D$. When we restrict the sheaf on $M \backslash D$ of the germs of primitives of $\Omega$ to a connected component $F$ of its total space, we obtain a Galois covering $\pi: F \rightarrow M \backslash D$ whose group $H$ is the group of periods of $\Omega$ (see for example [4]). We call it the multivalued primitive of $\Omega$. The elements $f_{x}$ of the fiber at $x$ are the determinations of $F$ at $x$. The map: $x \mapsto f_{x}(x)$ is single valued in the quotient $C / H$. We will identify it with $F$ (and will use the same notation). In this context, this allows us to introduce the fibers of $F$, to be those of the single valued associated map from $M \backslash D$ into $C / H$.

\section{Proposition 1.2. The statement of Theorem B implies that of Theorem A.}

Proof. Let $f_{1}^{\lambda_{1}} \cdots f_{p}^{\lambda_{p}}$ be defined on an open neighborhood $U$ of 0 in $\mathbb{C}^{n}$. We consider the desingularization map $E: M \rightarrow U$ of the analytic set $X$ with equation $f_{1} \cdots f_{p}=0$ given by [5]. Let $D=E^{-1}(X)$ and let $\Omega=E^{*} \sum_{i=1}^{p} \lambda_{i} \frac{d f_{i}}{f_{i}}$. We can easily check that $\Omega$ is logarithmic, and its residues are linear combinations of the $\lambda_{i}$ with positive integer coefficients [10]. So, they are non-zero, and their ratios are not negative real numbers. If $F$ is a primitive of $\sum_{i=1}^{p} \lambda_{i} \frac{d f_{i}}{f_{i}}$, then $F \circ E$ is a primitive of $\Omega$. It thus suffice now to consider the image of 
the neighborhoods of $D$ in $M$ given by Theorem $\mathrm{B}$ by the map $E$, to get neighborhoods of the singularity which satisfy the required property.

Remark 1.3. We apply here the desingularization theorem of an analytic set $X$ (imbedded in an analytic manifold $M$ ) in a local situation. So we can suppose - by choosing a good representant of the germ $X$ - that the singular locus of $X$ is connected. In particular, the exceptional divisor $D$ is also connected.

Remark 1.4. The second hypothesis of Theorem A can be improved: to apply Theorem B, it suffices to show that only the ratio of the residus of $\Omega=E^{*} \sum_{i=1}^{p} \lambda_{i} \frac{d f_{i}}{f_{i}}$ on irreducible components of $D$ with non-empty intersection are not negative real numbers.

Definition 1.5. Let $\Omega$ be a closed logarithmic one-form on $M$ with poles on $D$ and let $U$ be an open set of $M$. We will say that two points $P$ and $Q$ in $U \backslash D$ are $U$-equivalent (notation: $P \sim{ }_{U} Q$ ) if they belong to a same leaf of the restriction of the foliation defined by $\Omega$ in $U$.

To prove Theorem $\mathrm{B}$, we have to construct open neighborhoods $U$ of $D$ in $M$ such that for any pair of points $(P, Q)$ in $U \backslash D$ we gets that:

$$
(F(P)=F(Q) \text { in } C / H) \Rightarrow\left(P \sim_{U} Q\right) .
$$

\section{§2. Fibers of $\sum_{j=1}^{p} \lambda_{j} \log z_{j}$}

Let $\Omega$ be the logarithmic closed form $\sum_{j=1}^{p} \lambda_{j} \frac{d z_{j}}{z_{j}}$ and let $F$ be the primitive of $\Omega: \sum_{j=1}^{p} \lambda_{j} \log z_{j}$. The divisor $D$ is here the union of coordinate hyperplanes. The numbers $\lambda_{j}$ are assumed to be non-zero, without any supplementary hypothesis. For each $P$ in $D$, we denote: $I(P)=\{i \in\{1, \cdots, p\}$, $\left.P \in\left(z_{i}=0\right)\right\}$. For $P$ in $D \cap U$, we call the subspace of dimension $|I|=$ length of $I: z_{j}=z_{j}(P), j \notin I$ the transversal in $P$.

Proposition 2.1. Let $U$ be a polydisc centered at the origin of $\boldsymbol{C}^{p}$. Then $F^{-1}(c) \cap U$ is connected for any $c$ in $C / H$ such that $F^{-1}(c)$ meets $U$.

Proof. We first consider the fiber $F^{-1}(c)$ in $C^{* p}$ using the universal covering of $C^{* p}$ by the exponential of each coordinate. The form $\Omega$ can be lifted to 


$$
\Omega^{+}=\exp ^{p * \Omega}=\sum_{j=1}^{p} \lambda_{j} d y_{j}
$$

The leaves of $\Omega^{+}$are parallel hyperplanes which are coverings of the fibers of $F$ : the fiber $F^{-1}(c)$ is covered by the union of the hyperplanes $\sum_{j=1}^{p} \lambda_{j} y_{j}=d$, where $d$ runs over the class of the value $c$ in $C / H$. The restriction of this covering to one of these hyperplanes is still a (connected) covering of the whole fiber. So this fiber is connected in $\mathbb{C}^{* p}$. Furthermore we remark that this covering is trivial when there is no relation with integer coefficients between the $\lambda_{j}$. In this case, the fiber is homeomorphic to $C$.

Now let $U$ be a polydisc of $C^{p}$. It can be lifted to an intersection of half-spaces:

$$
\tilde{U}=\left\{\Re y_{j}<-A_{j}, j=1 \cdots p\right\},\left(A_{j} \text { large positive reals }\right)
$$

in which the trace of hyperplanes is still connected, and covers $F^{-1}(c) \cap U$. So the same is true for $F^{-1}(c) \cap U$.

Remark 2.2. Theorem $\mathrm{A}$ is proved in the particular case that $f$ is the exponential of a primitive of the form $\Omega=\sum_{j=1}^{p} \lambda_{j} \frac{d z_{j}}{z_{j}}$, for neighborhoods which are polydiscs. We remark that we have do not use the hypothesis $\lambda_{i} / \lambda_{j} \notin \mathbb{R}^{-}$ here. Nevertheless, this condition is necessary for the general case (see Remark (2.7) and a counter-example in last section).

To find the values $c$ for which $F^{-}(c)$ meets a given polydisc $U$, one has to distinguish two different geometric situations:

Proposition 2.3. Let $U$ be a polydisc centered at the origin of $\mathbb{C}^{p}$. We suppose that there exist two residues of $\Omega$ whose ratio is not a real number. Then for any $c$ in $C / H, D \cap U$ is contained in the closure of $F^{-1}(c) \cap U$.

From this statement, we immediately deduce (with the same hypothesis) that:

Corollary 2.4. 1. For any $c$ in $C / H, F^{-1}(c)$ meets $U$.

2. Let $\left(P, P^{\prime}\right)$ be a pair of in $D \cap U$ and let $\Delta, \Delta^{\prime}$ be two arbitrary small neighborhoods of $P$ and $P^{\prime}$ in their transversals. For each point $Q$ in $\Delta$, there exists a point $Q^{\prime}$ in $\Delta^{\prime}$ which is $U$-equivalent to $Q$.

Proof of Proposition (2.3). Suppose that the ratio of the residues $\lambda_{1}$ and $\lambda_{2}$ is not a real number. Then $\left(z_{1}=0\right) \cap U$ is in the closure of any fiber: 
indeed, on the transversal $z_{j}=z_{j}^{0}, j \neq 1$ to $\left(z_{1}=0\right) \cap U, F^{-1}(c)$ is defined by:

$$
\log z_{1}=\frac{c}{\lambda_{1}}+2 i \pi \sum_{j} n_{j} \frac{\lambda_{j}}{\lambda_{1}}-\sum_{j \neq 1} \frac{\lambda_{j}}{\lambda_{1}} \log z_{j}^{0}
$$

so

$$
\left|z_{1}\right|=\exp \Re\left(\frac{c}{\lambda_{1}}+2 i \pi \sum_{j} n_{j} \frac{\lambda_{j}}{\lambda_{1}}-\sum_{j \neq 1} \frac{\lambda_{j}}{\lambda_{1}} \log z_{j}^{0}\right) .
$$

The term $\exp -2 \pi n_{2} \mathfrak{I}\left(\lambda_{2} / \lambda_{1}\right)$, with $n_{2}$ an arbitrary integer and $\mathfrak{I}\left(\lambda_{2} / \lambda_{1}\right)$ =imaginary (non-vanishing) part of $\lambda_{2} / \lambda_{1}$, allows us to find points $P$ of $F^{-1}(c)$ with $\left|z_{1}(P)\right|$ as small as necessary. Because the inverse of $\lambda_{2} / \lambda_{1}$ is not a real number, $\left(z_{2}=0\right) \cap U$ is also in the closure of $F^{-1}(c)$. Given an arbitrary index $j$, different from 1,2 , one of the ratios $\lambda_{j} / \lambda_{1}$ or $\lambda_{j} / \lambda_{2}$ is not a real number. We deduce that $\left(z_{j}=0\right) \cap U$ is still in the closure of $F^{-1}(c)$.

Proposition 2.5. Let $U$ be a polydisc centered at the origin. Suppose that all the residues of $\Omega$ belong to a same real half-line, with direction $\lambda$.

1. There exists a neighborhood $V_{\varepsilon}$ of $D \cap U$ in $U$, saturated by the fibers of $F$ restricted to $U$.

2. Each fiber of $F$ in $V_{\varepsilon}$ meets every transversal at a point of $D \cap V_{\varepsilon}$.

Proof. When the residues $\lambda_{j}$ belong to the same real half-line $\lambda \boldsymbol{R}^{+}$, every point of $F^{-1}(c) \cap U$ satisfies:

$$
\sum_{j} \lambda_{j} \log \left|z_{j}\right|=\lambda \Re\left(\frac{c}{\lambda}\right)
$$

so, setting $\lambda_{j}=x_{j} \cdot \lambda$,

$$
\prod_{j}\left|z_{j}\right|^{x_{j}}=\exp \Re\left(\frac{c}{\lambda}\right) .
$$

Let $V_{\varepsilon}:\left(\prod\left|z_{j}\right|^{x_{j}}<\varepsilon\right) \cap U$. Because the exponents $x_{j}$ are positive, $V_{\varepsilon}$ is a neighborhood of $D \cap U$ in $U$, saturated for $\left.F\right|_{U}$.

Furthermore, if $z_{j}=z_{j}(M), j \geq 2$, the equation $\sum_{j} \lambda_{j} \log z_{j}=c$ with $\mathfrak{R}\left(\frac{c}{\lambda}\right)<\varepsilon$ always has solutions in the variable $z_{1}$ such that $\left(z_{1}, \cdots, z_{p}\right)$ belongs to $V_{\varepsilon}$. This proves (2).

Corollary 2.6. Suppose that all the residues $\lambda_{j}$ belong to the same real 
half-line $\lambda \boldsymbol{R}^{+}$. The neighborhood $V_{\varepsilon}$ constructed in Lemma (2.5) satisfies the following properties:

1. $F^{-1}(c) \cap V_{\varepsilon}$ is connected for every $c$ in $C / H$ such that $F^{-1}(c)$ meet $V_{\varepsilon}$.

2. Let $P$ and $P^{\prime}$ be two points of $D \cap U$, and let $\Delta, \Delta^{\prime}$ be the traces in $V_{\varepsilon}$ of transversals in $P$ and $P^{\prime}$. For every point $Q$ of $\Delta$, there exists a point $Q^{\prime}$ of $\Delta^{\prime}$ which is $V_{\varepsilon}$-equivalent to $Q$.

3. If $F^{-1}(c)$ meets $V_{\varepsilon}$ and $k$ is a "contracting" element of $C / H$ (that is to say $\left.\exp \mathfrak{R}\left(\frac{k}{\lambda}\right)<1\right)$ then $F^{-1}(c+k)$ also meets $V_{\varepsilon}$.

Proof. The first part is clear because $V_{\varepsilon}$ is a union of fibers of $F$ in $U$ which are connected according to the first part of (2.5). We deduce the second part from (2) of (2.5), and the last part is clear from the definition of $V_{\varepsilon}$.

Remark 2.7. It is important to note that property (2) in statements (2.4) and (2.6) is not satisfied if there exists a ratio of residues which is a negative real number. For this, it suffices to consider the function $x^{-\lambda} y$ where $\lambda$ is a positive real number. The fiber corresponding to the value 1 is contained in the real 3-manifold $|y|=|x|^{\lambda}$, which is not compatible with a property of type (2), if we choose a transversal on each component of $x y=0$.

Definition 2.8. The polydisc $U$, in the case where there exist two residues of $\Omega$ with a ratio non-real, and the saturated neighborhood $V_{\varepsilon}$ in the case where all the residues belong to the same real half-line, is called "neighborhood adapted" to $\Omega$.

Remark 2.9. The connectedness of the fibers for a primitive of $\Omega=\sum_{j=1}^{p} \lambda_{j} \frac{d z_{j}}{z_{j}}$ is proved in any neighborhood adapted to $\Omega$.

\section{§3. Fibers of a Primitive of a Closed Logarithmic Form}

We now consider the following data $(D, \Omega, F)$, where $\Omega$ is a closed logarithmic one-form on $M$ with poles on a normal crossing divisor $D$, and $F$ is a multivalued primitive of $\Omega$. We are only interested in the local situation along $D$. So we will assume that the group $H$ of periods of $\Omega$ only depends on the germ of $\Omega$ along $D$ : it is generated by the numbers $2 i \pi \mu_{j}$, where the $\mu_{j}$ are the residues of $\Omega$ around each component $D_{j}, j=1 \cdots q$ of $D$. For every point $P$ of $M$ and every open set $U$ of $M$ we define: 


$$
\begin{aligned}
& J(P)=\left\{j \in\{1, \cdots q\}, P \in D_{j}\right\} \\
& J(U)=\left\{j \in\{1, \cdots q\}, U \cap D_{j} \neq \emptyset\right\} .
\end{aligned}
$$

Definition 3.1. Let $P$ be a point of $D$, and let $S_{J}$ be the strata of $D$ which is the connected component of the set $\{Q \in D, J(Q)=J(P)\}$ containing $P$. A "local system of transverse coordinates adapted to $(D, \Omega, F)$ " in $P$ is a pair $\left(U_{\alpha}, z_{\alpha}^{J}\right)$, where $U_{\alpha}$ is a connected open set of $M$ containing $P$ such that $J\left(U_{\alpha}\right)=J$, and such that $z_{\alpha}^{J}$ is a map (analytic or $C^{\infty}$ ) from $U_{\alpha}$ to $C^{J}$ satisfying:

1. $D_{j} \cap U_{\alpha}$ is defined by the local equation: $z_{\alpha}^{j}=0$;

2. $\left.\Omega\right|_{U_{\alpha}}=z_{\alpha}^{J} * \Omega_{J}$, where $\Omega_{J}$ is the "model" logarithmic form on $C^{J}: \sum_{J} \mu_{j}$ $d z_{j} / z_{j}$

3. $\left.F\right|_{U_{\alpha} \backslash D} \equiv F_{J} \circ z_{\alpha}^{J}$, where $F_{J}$ is the multivalued primitive of the model: $\sum_{J} \mu_{j} \log z_{\alpha}^{j} . \quad$ Here the notation " $\equiv "$ means that the following diagram commutes:

$$
U_{\alpha} \backslash D \stackrel{F_{J} \circ z_{\alpha}^{J}}{\longrightarrow} \begin{gathered}
C / H_{J} \\
\downarrow \sigma_{J} \\
C / H
\end{gathered}
$$

where the group $H_{J}$ is the group of periods of $\Omega_{J}$, subgroup of $H$ generated by $\left\{2 i \pi \mu_{j}, j \in J\right\}$, and $\sigma_{J}$ is the canonical map from $\mathrm{C} / \mathrm{H}_{J}$ to $\mathrm{C} / \mathrm{H}$.

Definition 3.2. A "Clemens structure adapted to $(D, \Omega, F)$ " is a collection of tubular neighborhoods $\pi_{J}: T_{J} \rightarrow S_{J}$ over each strata $S_{J}$ of $D$ such that:

1. The local trivializations $z_{\alpha}^{J}: \pi_{J}^{-1}\left(V_{\alpha}\right) \rightarrow C^{J}$ are transverse coordinates $C^{\infty}$ adapted to $(D, \Omega, F)$.

2. For any pair $\left(S_{I}, S_{J}\right)$ of incident strata $\left(S_{I}\right.$ contained in the closure of $\left.S_{J}\right)$, we have:

(a) an inclusion of fibers: for any $P$ in $U_{\beta} \subset S_{J} \cap T_{I}$ we have that

$$
\pi_{J}^{-1}(P)=\pi_{I}^{-1}\left(\pi_{I}(P)\right) \bigcap\left(\cap\left(z_{\beta}^{i}=z_{\beta}^{i}(P), i \in I-J\right)\right.
$$

(b) compatibility between local coordinates: for open sets $V_{\alpha}$ in $S_{J}$ and $V_{\beta}$ in $S_{I}$ such that $V_{\alpha} \subset \pi_{I}^{-1}\left(V_{\beta}\right) \cap S_{J}$ we have that

$$
z_{\alpha}^{j}=z_{\beta}^{j} \cdot \exp \sum_{i \in I-J} \frac{\mu_{i}}{|J| \mu_{j}} \log z_{\beta}^{i}, \quad \text { for all } j \text { in } J
$$

The existence of such a structure is proved constructively in [9]. It is easy 
to prove the existence of local transverse coordinates adapted to $(D, \Omega, F)$, and also to construct tubular neighborhoods adapted along each strata. The main difficulty is to construct them with the compatibility conditions of (2). This can only be done in the $C^{\infty}$ class of differentiability.

Remark 3.3. From formula (b), for every $J \subset I$ we obtain that:

$$
F_{I} \circ z_{\beta}^{I}=\sigma_{I J} \circ F_{J} \circ z_{\alpha}^{J},
$$

where $\sigma_{I J}$ is the canonical map from $\mathbb{C} / H_{J}$ to $\mathbb{C} / H_{I}$.

Defimition 3.4. An open set $U$ of $M$ is a "good open set" for $\Omega$ if it satisfies:

1. for any point $P$ of $D$ such that $U \cap \pi_{I}^{-1}(P) \neq \emptyset$, the image of $U \cap \pi_{I}^{-1}(P)$ in $\mathbb{C}^{I}$ by a local transverse coordinate adapted to $(D, \Omega, F)$ is a neighborhood adapted to $\Omega_{I}(2.8)$.

2. For any pair of points $\left(P, P^{\prime}\right)$ in $D \cap U$, where $P$ is in a strata $S_{J}$ and $P^{\prime}$ is in $S_{K}$, and for any point $Q$ over $P$ (i.e. which belongs to $\left.\pi_{J}^{-1}(P) \cap U\right)$, there exists a point $Q^{\prime}$ over $P^{\prime}$ (i.e. on $\left.\pi_{K}^{-1}\left(P^{\prime}\right) \cap U\right)$ which is U-equivalent to $Q$.

A neighborhood of $D$ (or a subset of $D$ ) in $M$ is a "good neighborhood" for $\Omega$ when it contains a good open set covering $D$ (or this subset of D).

In other terms, in a good neighborhood, every leaf can be seen everywhere: it meets any transversal to the exceptional divisor. The proof of Theorem $B$ is a consequence of the following two statements:

Proposition 3.5. Let $\Omega$ be a closed logarithmic one-form with non-vanishing residues. If the ratio of residues on two components with non-empty intersection are not negative real numbers, then there exists a fundamental system of good neighborhoods of $D$ in $M$ for $\Omega$.

Proposition 3.6. Let $U$ be a good neighborhood for $\Omega$. For any pair of points $(P, Q)$ in $U \backslash D$ we have that

$$
(F(P)=F(Q) \text { in } C / H) \Rightarrow\left(P \sim_{U} Q\right)
$$

Proof of the Proposition (3.5). We split it into two parts.

First step. Let $S_{J}$ be a strata of $D$ and let $S_{J}^{\prime}$ be an open set in $S_{J}$, with 
compact closure in $S_{J}$. We shall prove that there exists a good neighborhood $U_{J}$ of $S_{J}^{\prime}$ in $M$. For this, take a base point $P_{0}$ in $S_{J}^{\prime}$. For any point $P$ of $S_{J}^{\prime}$, there exists an open set containing $P_{0}$ and $P$, over which the foliation defined by $\Omega$ is trivial. We consider a covering of $S_{J}^{\prime}$ by such open sets, and extract from it a finite covering $\left\{V_{\alpha}, \alpha \in \Lambda\right\}$. Let $z_{\alpha}: \pi_{J}^{-1}\left(V_{\alpha}\right) \rightarrow C^{J}$ be an adapted transverse coordinate over $V_{\alpha}$. Choose an adapted neighborhood $\Delta_{J}$ in $C^{J}$ contained in the intersection of the images of $z_{\alpha}, \alpha \in \Lambda$. Let $U_{\alpha}$ be the open set of $M$ which is the inverse image by $z_{\alpha}$ of $\Delta_{J}$ and let $U_{J}$ be the union of the $U_{\alpha}, \alpha$ in $\Lambda$. By construction, $U_{J}$ satisfies the first condition of Definition (3.4). On the other hand, by triviality of the foliation in $V_{\alpha}$, for any point $Q$ of $V_{\alpha}$ over a point $P$ of $U_{\alpha}$, there exists a point $Q_{0}$ over $P_{0}$ which is $U_{\alpha^{-}}$and so $U_{J}$-equivalent to $Q$. Because $z_{\alpha}\left(Q_{0}\right)$ belongs to $\Delta_{J}=z_{\beta}\left(U_{\beta}\right)$, for every $P^{\prime}$ of $V_{\beta}$ there exists $Q^{\prime}$ in $U_{\beta}$ which is $U_{J}$-equivalent to $Q_{0}$. By transitivity, we get condition 2) of definition (3.4): $U_{J}$ is a good open set for $\Omega$.

Second step. For convenience, we will say that a strata $S_{I}$ is "real" if all the ratios of the residues related to it are positive real numbers. It is "non-real" if there exist a ratio of residues which is non-real. We choose the open sets $S_{J}^{\prime}$ large enough so that the union $U$ of the open sets $U_{J}$ constructed in the previous step give us a neighborhood of $D$ in $M$. We require more: let $S_{J}$ be a strata contained in the closure of $S_{I}$.

- if $S_{J}$ (and a fortiori $S_{I}$ ) is real, we note that from the change of coordinates formulae between the strata of a Clemens structure, an adapted neighborhood $V_{\varepsilon}^{J}$ for transverse coordinates on $S_{J}$ is still an adapted neighborhood $V_{\varepsilon}^{J}$ for transverse coordinates to $S_{I}$. We will ensure that an appropriate $\varepsilon$ is chosen for incident real strata.

- if $S_{J}$ is non-real, we will only require that $U_{J}$ is large enough to get, for every point $P$ in $U_{J} \cap S_{I}$, the inclusion of $\pi_{I}^{-1}(P)$ in $U_{J}$.

In order to prove that $U$ is a good neighborhood, it suffices to check the following property: let $S_{K}$ and $S_{I}$ be two strata whose closures have non-empty intersection. For any pair of points $\left(P, P^{\prime}\right)$ in $D, P$ in $S_{I}, P^{\prime}$ in $S_{K}$, and for any point $Q$ in $\pi_{I}^{-1}(P) \cap U_{I}$, there exists a point $Q^{\prime}$ in $\pi_{K}^{-1}\left(P^{\prime}\right) \cap U_{K}$, which is $U$-equivalent to $Q$. For this, consider a strata $S_{J}$ contained in the intersection of the closure of $S_{K}$ and of $S_{I}$. Let $U_{J}$ be the open set related to $S_{J}$ by the construction of the previous step. We may suppose - by exchanging $P$ with a $U_{I}$-equivalent point - that $P$ belongs to $S_{I} \cap U_{J}$. Similarly, we may suppose that $P^{\prime}$ belongs to $S_{K} \cap U_{J}$. Furthermore, from the first step (applied to $U_{J}$ ), we may suppose that $P$ and $P^{\prime}$ are on the same fiber $\pi_{J}^{-1}(R)$, of the 
tubular neighborhood of $S_{J}$. Using Definition (3.2, 2-) the fibers $\pi_{I}^{-1}(P)$ and $\pi_{K}^{-1}\left(P^{\prime}\right)$ are contained in $\pi_{J}^{-1}(R)$, and are defined in adapted transverse coordinates $\left(z_{j}, j \in J=I \cup K\right)$ over $S_{J}$ by

$$
\begin{gathered}
z_{k}=z_{k}(P), \quad k \in K, \\
z_{i}=z_{i}\left(P^{\prime}\right), \quad i \in I .
\end{gathered}
$$

We are now, via the trivialization $z_{J}$ in the situation that we consider in (2.4, 2-) if $S_{J}$ is non-real, or in $(2.6,2-)$ if it is real.

Proof of the Proposition (3.6). Let $U$ be a good neighborhood of $D$ and let $P_{0}, Q_{0}$ be two points of $U \backslash D$ on the same fiber of a primitive $F$ of $\Omega: F\left(P_{0}\right)=F\left(Q_{0}\right)$ in $C / H$. We have to prove that $P_{0}$ and $Q_{0}$ are $U$ equivalent. Because $U$ is a good open set, we can suppose — by exchanging $Q_{0}$ with a $U$-equivalent point - that $P_{0}$ and $Q_{0}$ are over the same point $M_{0}$ in a strata $S_{I}$ of $D$. Let $\left(U_{\alpha}, z_{\alpha}^{I}\right)$ be a local system of adapted transverse coordinates in $M_{0}$. By (3.1), we see that

$$
F_{I} \circ z_{\alpha}^{I}\left(P_{0}\right)=F_{I} \circ z_{\alpha}^{I}\left(Q_{0}\right)+\sum_{j \neq I} 2 i \pi n_{j} \mu_{j} \quad \text { in } C / H_{I}
$$

If $S_{I}$ is a real strata, we make sure that the constants are distributed amongst the two appropriate terms in the equation so they are all contracting (see 3- of (2.6)). We get that

$$
F_{I} \circ z_{\alpha}^{I}\left(P_{0}\right)+\sum_{j \notin I} 2 i \pi n_{j} \mu_{j}=F_{I} \circ z_{\alpha}^{I}\left(Q_{0}\right)+\sum_{k \notin I} 2 i \pi n_{k} \mu_{k} \quad \text { in } \quad C / H_{I}
$$

Remark 3.7. If in equation (2), all the integers $n_{j}$ and $n_{k}$ are equal to zero, then $P_{0}$ and $Q_{0}$ are in the same "local" fiber of $F_{I}$. We conclude that they are $U$-equivalent, using property (1) of a good neighborhood (3.4) and Remark (2.9).

Our strategy is to reduce to this situation, using the following lemma:

Lemma 3.8. Let $2 i \pi n_{j} \mu_{j}$ be a contracting element of $C / H_{I}$. There exists a point $P_{1}$ on the transverse fiber $\pi_{I}^{-1}\left(M_{0}\right) \cap U$ which is $U$-eqivalent to $P_{0}$, such that:

$$
F_{I} \circ z_{\alpha}^{I}\left(P_{1}\right)=F_{I} \circ z_{\alpha}^{I}\left(P_{0}\right)+2 i \pi n_{j} \mu_{j} \text { in } C / H_{I} .
$$


Proof. Let $c_{0}$ be the element of $C / H_{I}$ defined by $F_{I} \circ z_{\alpha}^{I}\left(P_{0}\right)=c_{0}$. The local level hypersurface

$$
\left(F_{I} \circ z_{\alpha}^{I}\right)^{-1}\left(c_{0}+2 i \pi n_{j} \mu_{j}\right)
$$

also meets $\pi_{I}^{-1}\left(M_{0}\right) \cap U$, either because its closure contains the strata (2.4), or if $S_{I}$ is real, because $2 i \pi n_{j} \mu_{j}$ is contracting $(2.6,3-)$. Let $P_{1}$ be a point in this intersection. By construction, it satisfies the equation (3). We have only to prove that $P_{1}$ is $U$-equivalent to $P_{0}$. This is easy in the following case:

First case. Suppose that $j$ is an "indice near $I$ ", that is to say, $j$ belongs to $J \supset I$, with $S_{J} \subset \overline{S_{I}}$. Because $U$ is good neighborhood, we may suppose - by exchanging $P_{0}$ and $P_{1}$ with $U$-equivalents points - that $P_{0}$ and $P_{1}$ are over the same point of $S_{I} \cap T_{J}$. When we move them over the same path - which is allowed by the construction of the good open sets $U_{I}$-equation (3) is invariant. When we look at it in coordinates adapted to $S_{J}$, it becomes, from (3.3):

$$
F_{J} \circ z_{\alpha}^{J}\left(P_{1}\right)=F_{J} \circ z_{\alpha}^{J}\left(P_{0}\right)+0 \text { in } C / H_{J} .
$$

Then we are in the situation of Remark (3.7): $P_{0}$ and $P_{1}$ are $U$-equivalent. The lemma is thus proved in this particular case.

General case. We construct a graph associated to the stratification of $D$ in the following way: the vertices are strata of $D$. They are joined by a branch when the relation "is incident to" is satisfied. We put all the strata with the same codimension on the same horizontal line:

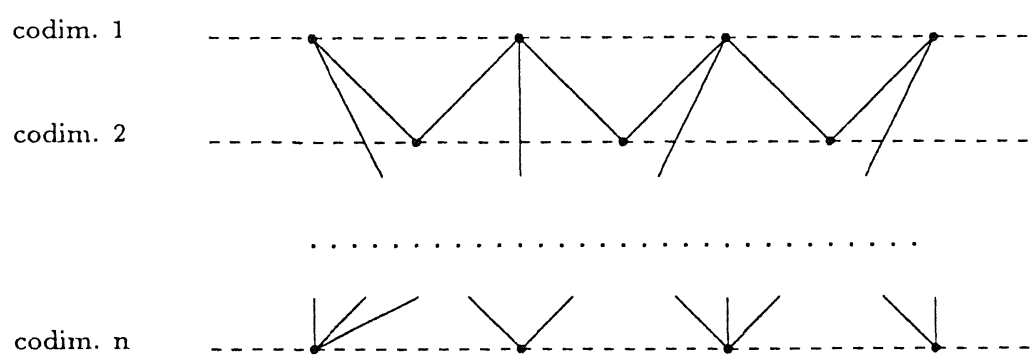

This graph is connected, because $D$ is connected by Remark (1.3). Let $S_{J}$ be a 
strata of $D$ such that $J$ contain the indice $j$ of the contracting constant in equation (3). We choose a path joining the vertices $S_{I}$ and $S_{J}$. Property (1) of the definition of a good neighborhood allows us to move the points $P_{0}$ and $P_{1}$ to two $U$-equivalent other points which lie over any given point $M$ of $D$. For convenience, we will also call these points $P_{0}$ and $P_{1}$. We now move the point $M$ along a path in $D$ which join $S_{I}$ and $S_{J}$ in the graph. During the first change of strata from $S_{I}$ to a neighboring strata $S_{K}$, two different situations can occur:

- either we go from the strata $S_{I}$ to $S_{K}$ going down the graph $(I \subset K)$. The codimension of the strata increases. In this case, using formula (3.3) and applying the map $\sigma_{K I}$ to each term of equation (3), we get that:

$$
F_{K} \circ z_{\alpha}^{K}\left(P_{1}\right)=F_{K} \circ z_{\alpha}^{K}\left(P_{0}\right)+2 i \pi n_{j} \mu_{j} \text { in } C / H_{K} .
$$

- or we go up the graph. In this case, the operation by which we obtain $U$-equivalent points may be achieved by taking a section of $\sigma_{I K}$ for each term in the equation. A consequence of this indeterminacy is the appearance of a new constant $2 i \pi n_{i} \mu_{i}$ in (3), which is in the kernel of $\sigma_{I K}$, that is to say in $H_{I} / H_{K}$. We get that:

$$
F_{K} \circ z_{\alpha}^{K}\left(P_{1}\right)=F_{K} \circ z_{\alpha}^{K}\left(P_{0}\right)+2 i \pi n_{j} \mu_{j}+2 i \pi n_{i} \mu_{i} \text { in } \mathbb{C} / H_{K} .
$$

We remark then that this new indice $i$ is a "indice near $K$ ": we can use the first case to make this constant disappear, by exchanging $P_{0}$ with a $U$-equivalent point. If, in the "real case", $2 i \pi n_{j} \mu_{j}$ is not contracting, we will change its term, and will exchange the other point $P_{1}$ with a $U$-equivalent point. This manipulation ensures that every new constant which may appear when we go up the graph will cancel. At the end of the path, we get the following eguation:

$$
F_{J} \circ z_{\alpha}^{J}\left(P_{1}\right)=F_{J} \circ z_{\alpha}^{J}\left(P_{0}\right)+2 i \pi n_{j} \mu_{j} \text { in } C / H_{J} .
$$

in which $2 i \pi n_{j} \mu_{j}$ is equal to zero in $C / H_{J}$. We are now in the situation of Remark (3.7). So $P_{0}$ and $P_{1}$ are $U$-equivalent.

End of the proof of the Proposition (3.6). Consider the equation (2). Iterating Lemma (3.8), we construct two finite sequences of points $\left\{\boldsymbol{P}_{j}\right.$, $j=0, \cdots, r\}$ and $\left\{Q_{k}, k=0, \cdots, s\right\}$ with $P_{j} \sim_{U} P_{0}$ and $Q_{k} \sim_{U} Q_{0}$ such that:

$$
F_{I} \circ z_{\alpha}^{I}\left(P_{r}\right)=F_{I} \circ z_{\alpha}^{I}\left(P_{0}\right)+\sum_{j \notin I} 2 i \pi n_{j} \mu_{j} \text { in } C / H_{I}
$$




$$
F_{I} \circ z_{\alpha}^{I}\left(Q_{s}\right)=F_{I} \circ z_{\alpha}^{I}\left(Q_{0}\right)+\sum_{k \notin I} 2 i \pi n_{k} \mu_{k} \text { in } C / H_{I}
$$

Equation (2) is equivalent to

$$
F_{I} \circ z_{\alpha}^{I}\left(P_{r}\right)=F_{I} \circ z_{\alpha}^{I}\left(Q_{s}\right) \text { in } C / H_{I}
$$

which, from Remark (3.7), shows that: $P_{r} \sim U Q_{s}$, and so $P_{0} \sim U Q_{0}$.

\section{§. Counter-examples}

Consider the family of functions at the origin of $C^{2}$

$$
f_{\alpha}=\left(x^{2}-y^{3}\right)\left(x^{3}-y^{2}\right)^{-\alpha}
$$

where the real parameter $\alpha$ satisfies $1<\alpha<\frac{3}{2}$. Let us now give a brief description of the fibers of $f$. For more details, see [11]. The desingularization of the closed logarithmic form $d f / f$ can be represented by the following tree:

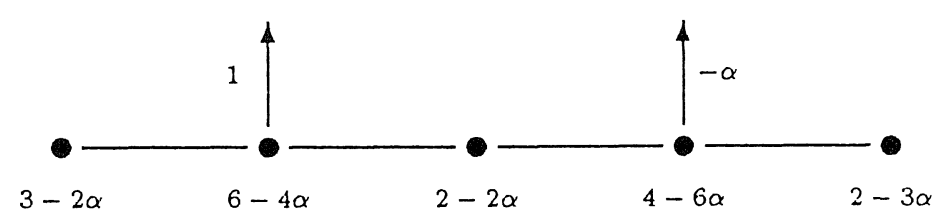

The vertices represent irreducible components of the exceptional divisor. The coefficients $\lambda$ are the "multiplicities" of $f$ on each component: $2 i \pi \lambda$ is the residue on this component of the inverse image of $d f / f$ by the desingularization map. Two vertices are joined if the intersection of the corresponding components is non-empty. The arrows represent strict inverse images of each branch of $\left(x^{2}-y^{3}\right)\left(x^{3}-y^{2}\right)$. So the second vertex represents the "corner" intersection of the component with multiplicity $6-4 \alpha$ and that of $2-2 \alpha$. From the choice of the interval of definition of $\alpha$, the ratio of these two multiplicities is a non-zero negative real number.

We investigate the local trace of the fiber $\log f=c$ ( $c$ is in the quotient of $C$ by the additive group $(1,-\alpha)$ generated by $2 i \pi$ and $2 i \pi \alpha)$, in a neighborhood of a regular point of the component with multiplicity $6-4 \alpha$. It is given by: 


$$
(6-4 \alpha) \log z=c+h, \quad h \in(1,-\alpha)
$$

and it intersects a disc $|z|<r$ at points with coordinates

$$
z=\exp \left(c+\frac{h}{6-4 \alpha}\right), \quad h \in(1,-\alpha)
$$

So there is an injection from this local image of $\log f=c$ into the quotient $(1,-\alpha) /(6-4 \alpha)$.

"Semi-locally", that is to say on an adapted neighborhood of the smooth part of this component, the fiber has two connected components. Indeed, a pair of local connected components are in the same-locally connected component if, transported close to another component, they can be glued into a connected component of the fiber in the neighborhood of the corner. In our case, this condition is satisfied if their corresponding values by the previous injection are the same in the quotient of $(1,-\alpha) /(6-4 \alpha)$ by the residues of the neighboring components:

$$
(1,-\alpha) /(6-4 \alpha, 3-2 \alpha, 1,2-2 \alpha)
$$

This quotient is also equal to

$$
(1,-\alpha) /(1,-2 \alpha)
$$

which contains only two elements (the classes of 0 and $-\alpha$ ). These two components are non-empty in arbitrarily small neighborhoods: we can find local representatives $z=\exp \left(c+\frac{h}{6-4 \alpha}\right)$ of each of them with $|z|$ arbitrarily small.

If we now investigate this fiber in an adapted neighborhood of the union of this component with the "end-component" (with multiplicity $3-2 \alpha$ ), it still has two connected components: topologically, the fiber over the smooth part of this end-component is a union of discs which will block corresponding holes in the fiber over the corner.

Let $C$ be the component of the exceptional divisor corresponding to the central vertex, and let $C^{\prime}$ be equal to $C$ minus small discs around the two corners. From Remark (2.7), we notice that for any transversal at a regular point of $C$ with small enough height, the two connected components of the fiber over the left part of the tree do not meet this transversal. So there exists a neighborhood $U$ of $C^{\prime}$ whose intersection with these two connected components is empty. In any neighborhood of the whole divisor thin enough to get a trace over $C^{\prime}$ contained in $U$, the fiber will have at least two connected 
components.

Notice that the element $f_{4 / 3}$ belongs to this family of counterexamples. This element defines the same foliation as the meromorphic function

$$
\left(x^{2}-y^{3}\right)^{3}\left(x^{3}-y^{2}\right)^{-4} \text {. }
$$

So this function does not satisfy the connectedness property of Theorem A. Nevertheless, there exist meromorphic functions which satisfy this property: the local models $x^{p} y^{-q}$ with $p$ and $q$ positive integers, which are not a power $($ g.c.d. $(p, q)=1)$ are examples of this class, from (2.2).

At the endpoint of this family of counter-examples, the meromorphic function $f_{1}$ is interesting. In this case, the hypothesis of Theorem $\mathrm{A}$ is violated strongly: the central residue is equal to zero. The corresponding component of the divisor is dicritical: after reduction, the foliation is transverse to this component. It is also a "cut" for the left and right sides of the tree. This cut phenomenon does not occur for the functions $x^{p} y^{-q}$ because the dicritical component is an endpoint of the tree.

\section{References}

[1] Briançon, J., Galligo, A. and Granger, M., Déformations équisingulières des germes de courbes gauches réduites, Mém. Soc. Math. France, 108-1 (1980-1981).

[2] Camacho, C., Neto, A. Lins and Sad. P., Topological invariants and equidesingularization for holomorphic vector fields, J. Differential Geom., 20 (1984), 143-174.

[3] Cerveau, D. and Mattei, J. F., Formes intégrables holomorphes singulières, Astérisque, 97, (1982), 1-192.

[4] Godbillon, C., Eléments de Topologie algébrique, Hermann, Collection Méthodes, 1971.

[5] Hironaka, H., Introduction to the theory of infinitely near singular points, Memorias de Matematica del Instituto Jorge Juan Madrid, 28, 1974.

[6] Kabila, A., Sur les feuilletages logarithmiques, C.R. Acad. Sc. Paris, 302 (1986), 13-15.

[7] Lê D. T., Calcul du nombre de cycles évanouissants d'une hypersurface complexe, Ann. Inst. Fourier, 23, 4 (1973), 261-270.

[8] Mattei, J. F. and Moussu, R., Holonomie et intégrales premières, Ann. Sci. Ecole Norm. Sup., 13, (1980), 571-621.

[9] Paul, E., Etude topologique des formes logarithmiques fermées, Invent. Math., 95 (1989), 395-420.

[10] Classification topologique des germes de formes logarithmiques génériques, Ann. Inst. Fourier, 39-4 (1989), 909-927.

[11] $\longrightarrow$ Cycles évanescents d'une fonction de Liouville de type $f_{1}^{\lambda_{1}} \cdots f_{p}^{\lambda_{p}}$, Ann. Inst. Fourier, 45-1 (1995), 31-63.

[12] - Formes singulières à holonomie résoluble, Prépublication de Laboratoire E. Picard (1995).

[13] Saito, K., Theory of logarithmic differential forms and logarithmic vector fields, J. Fac. Sci. Univ. Tokyo, 27 (1980), 265-291. 
